\title{
Excitonic effects in absorption spectra of carbon dioxide reduction photocatalysts
}

\author{
Tathagata Biswas ${ }^{1}$ and Arunima K. Singh iD $^{1 凶}$
}

The formation and disassociation of excitons play a crucial role in any photovoltaic or photocatalytic application. However, excitonic effects are seldom considered in materials discovery studies due to the monumental computational cost associated with the examination of these properties. Here, we study the excitonic properties of nearly 50 photocatalysts using state-of-the-art Bethe-Salpeter formalism. These $\sim 50$ materials were recently recognized as promising photocatalysts for $\mathrm{CO}_{2}$ reduction through a data-driven screening of 68,860 materials. Here, we propose three screening criteria based on the optical properties of these materials, taking excitonic effects into account, to further down select six materials. Furthermore, we study the correlation between the exciton binding energies obtained from the Bethe-Salpeter formalism and those obtained from the computationally much lessexpensive Wannier-Mott model for these chemically diverse $\sim 50$ materials. This work presents a paradigm towards the inclusion of excitonic effects in future materials discovery for solar-energy harvesting applications.

npj Computational Materials (2021)7:189; https://doi.org/10.1038/s41524-021-00640-3

\section{INTRODUCTION}

Excitons, which are quasiparticles, form when light is absorbed by semiconductors or insulators. They consist of a photoexcited electron and a hole bound by Coulomb interaction. In photocatalytic and photovoltaic processes these excitons must dissociate into free carriers and eventually reach the reacting species (in a photocatalytic process) or get converted into photocurrent (in a photovoltaic device) before any recombination processes can occur. Spontaneous generation of electron-hole $(e-h)$ pairs from the dissociation of excitons is only possible when exciton binding energy (EBE) is lower than $T \Delta S_{\text {diss, }}$ where $T$ is the environmental temperature and $\Delta S_{\text {diss }}$ is the entropy increase upon dissociation ${ }^{1}$. In a system where the number of photogenerated excitons $\left(n_{\mathrm{x}}\right)$ and the number of dissociated free e-h pairs $\left(n_{\mathrm{c}}=n_{\mathrm{e}}=n_{\mathrm{h}}\right)$ attains quasi-equilibrium, they are related $\mathrm{as}^{2}$,

$n_{\mathrm{c}}^{2}=n_{\mathrm{x}} T^{3 / 2} \exp \left(-E_{\mathrm{b}} / 2 k_{\mathrm{B}} T\right)$

where $T$ is the temperature, $E_{\mathrm{b}}$ is the $\mathrm{EBE}$, and $k_{\mathrm{B}}$ is the Boltzmann constant. Therefore, if everything remains the same, by increasing the EBE by $50 \mathrm{meV}$ the number of available free carriers $\left(n_{\mathrm{c}}\right)$ will be reduced by a factor of $\sim 2.6$ at $298 \mathrm{~K}$. This reduction in $n_{\mathrm{c}}$ directly affects the reaction rate of a photoreduction process. Once free $\mathrm{e}-\mathrm{h}$ pairs are available, a low carrier recombination rate is desirable as it ensures most of these $\mathrm{e}-\mathrm{h}$ pairs can be utilized in photocatalytic/photovoltaic applications. Both radiative, which are predominant in direct gap semiconductors, as well as nonradiative recombination processes have adverse effects on the quantum efficiency of a photocatalytic/photovoltaic device. However, with the help of innovative solutions such as use of multi-junction photocatalysts or solar cells ${ }^{3,4}$ one can go beyond the limit set by radiative recombination processes. In the context of solar cells, this limit is given by the Shockley-Queisser limit $^{5}$ and a lower limit is expected for photocatalysts ${ }^{6}$. Non-radiative recombinations that fall in the category of Shockley-Read-Hall ${ }^{7}$ or trap-assisted recombinations can also be controlled by improving device quality and using defect-free semiconductors ${ }^{8}$. The other kind of non-radiative recombination, known as Auger recombination $^{9,10}$ is quite hard to eliminate ${ }^{11}$. High EBE is detrimental for a photovoltaic/photocatalytic device as it can facilitate these Auger recombination processes ${ }^{10,12}$. Although, qualitatively it is obvious that a higher EBE will increase the Auger recombination rate $\left(R_{\text {Auger }}\right)$, we could not find any study that explicitly calculates this functional dependence in case of 3-dimensional semiconductors. However, in 1-dimensional systems it has been shown ${ }^{13}$ that the Auger recombination rate $R_{\text {Auger }} \propto \mathrm{EBE}^{3}$. Therefore, it is important to consider excitonic effects while looking for promising photovoltaics and photocatalysts.

Excitonic effects have been seldom considered in material discovery studies conducted in the past for identifying promising photovoltaic or photocatalytic materials ${ }^{14-18}$. For instance, recently, Singh et al. ${ }^{18}$ performed the largest $\mathrm{CO}_{2}$ photocathode search to date, where they shortlisted 52 materials starting from a list of 68,860 candidate materials. Their first-principles computation-based screening strategy was based on evaluating the thermodynamic stability, the electrochemical stability ${ }^{19}$, and the electronic structure compatibility with the $\mathrm{CO}_{2}$ reduction reaction $^{18}$. Their screening criteria were highly selective of robust photocatalysts when applied to the scores of known photocatalysts. It is noteworthy that the 52 materials shortlisted by Singh et al. were selected based on their (photo)electrochemical stability in the harsh reducing conditions needed for $\mathrm{CO}_{2}$ reduction reactions ${ }^{18,19}$. However, despite its success, the screening did not incorporate any excitonic effects. In recent years, Bethe-Salpeter formalism (BSE) has gained remarkable success in probing excitonic effects in bulk ${ }^{20-22}$ as well as 2-dimensional materials ${ }^{23-25}$, where these effects are more pronounced. The BSE formalism has a high computing resource requirement, both in terms of CPU time and memory. But, it has been found to have excellent agreement with experimental measurements across various materials classes ${ }^{20-25}$.

Most of the $\mathrm{CO}_{2}$ reduction photocatalysts available today such as $\mathrm{TiO}_{2}$ (probably the most studied material in this context) are wide bandgap semiconductors that are most efficient under

\footnotetext{
${ }^{1}$ Department of Physics, Arizona State University, Tempe, AZ 85287, USA. ${ }^{\circledR}$ email: arunimasingh@asu.edu
} 
UV radiation and can utilize only a negligible fraction of solar radiation $(5 \%)^{26}$. To enhance the visible light $(53 \%$ of solar radiation at the sea level) response of candidate photocatalytic materials, and thus utilize the major fraction of the solar energy, the search for promising materials for $\mathrm{CO}_{2}$ reduction photocatalysts should assess the optical response of these materials. Our study's central goal is to assess the feasibility of absorbing visiblelight through the computation of the entire frequency dependent absorption spectra of materials as opposed to looking at only the bandgap of materials as done in several other similar highthroughput studies done in the past ${ }^{27,28}$.

In this study, we design a materials screening strategy which is based on the optical properties and considers excitonic effects to identify the most efficient photocatalysts for $\mathrm{CO}_{2}$ reduction among the 52 materials shortlisted by Singh et al. We employ many-body perturbation theory within GW approximation together with ${ }^{29-31}$ BSE to explore quasiparticle and optical properties. Our screening strategy involves three distinct properties-EBE of the materials, the capability to absorb incident (visible or UV) radiation, and the degree of anisotropy in absorbing light of different polarizations. For each of these properties, we propose the limiting value for the criteria by looking at some of the well-known photocatalysts used for $\mathrm{CO}_{2}$ reduction. Using these criteria we identify a shortlist of six suitable materials from Singh et al.'s study that display optical properties which are at par or better than known photocatalysts. This screening approach is broadly applicable to the discovery of materials for other solar-energy applications. The limiting value corresponding to each of the properties in such a screening strategy will, however, change based on the specific application.

As the BSE is computationally resource intensive and timeconsuming, we also assess the prospect of using the computationally much less demanding Wannier-Mott model to compute the EBE of materials. We compare the EBE obtained from the BSE method and the Wannier-Mott (WM) model for 48 materials from Singh et al.'s study. These materials are chemically diversecomprising of oxides, sulfides, tellurides, and arsenides. We find that the WM model is effective in classifying low and high EBE materials. Thus, we surmise that the WM model can be used in place of the BSE method to identify materials with low EBE in a computational cheaper, hence potentially a high-throughput manner, to enable computational data-driven materials discovery for applications involving solar-absorption such as photovoltaics and photocatalysis.

\section{RESULTS AND DISCUSSION}

\section{Comparison between GW-BSE and HSE results}

Singh et al. ${ }^{18}$ had employed density-functional theory (DFT) with a hybrid exchange-correlation functional to compute the bandgaps and band edges of potential photocatalysts and identified materials that can utilize the visible-light spectrum which accounts for $44 \%$ of the solar radiation and simultaneously facilitate the reduction reaction of $\mathrm{CO}_{2}$. DFT which is designed to explore ground state properties suffers from bandgap underestimation problem as the bandgap is an excited state property of materials. Hybrid functionals such as HSE and HSE06, overcome this issue by adding a fraction (a) of Hartree-Fock exchange to the traditional exchange-correlation potential (LDA or GGA) and is quite successful in predicting correct bandgaps of a wide variety of materials. While hybrid functionals typically exhibit improved treatment of semiconductor bandgaps, they are heavily reliant on the fraction of the exact exchange $(a)$ included in the functional and are thus limited in their predictive capacity ${ }^{32}$. Once the accurate bandstructure is procured, although TDHSE can capture excitonic effects like BSE, solving the Cassida's Equation is equally expensive ${ }^{33}$. Moreover, earlier studies which employed TDHSE in probing excitonic effects seem to always underestimate EBE (often by an order of magnitude) ${ }^{34}$. Our TDHSE calculations (available in the Supplementary Table 7 and Supplementary Fig. 2) for AlSb, $\mathrm{TiO}_{2}$, and $\mathrm{MoSe}_{2}$ also draw similar conclusions.

Many-body perturbation theory within GW approximation is often an order of magnitude more expensive than standard DFT with hybrid functionals but does not require any empirical parameters and is found to be remarkably successful in case of various materials classes. The GW formalism combined with BSE has been proven to be the state-of-the-art method to study the electronic structure and optical properties of a wide variety of semiconductor and insulator materials in recent years with an excellent predictive capacity ${ }^{35-37}$. Moreover, the GW-BSE computed absorption spectra have very good agreement with experimentally measured spectra. Thus, we reevaluate the bandgaps of 48 photocatalysts from Singh et al.'s study using the GW method. We have restricted our study to the nonmagnetic 48 of the 52 materials identified by Singh et al. We exclude the $Z V F_{6}$ and the two phases of VOF as they have a magnetic ground state and the $\mathrm{CdHgAsBr}$ since it was found to be metallic according to our GW calculations.

Figure 1 shows the difference between the HSE06 bandgaps, $E_{\mathrm{g}}^{\mathrm{HSE}}$, reported in Singh et al.'s work and the quasiparticle (QP) gaps obtained from $G_{0} W_{0}, E_{g}^{G_{0} W_{0}}$, as well as the optical gaps obtained from $\mathrm{G}_{0} \mathrm{~W}_{0}-\mathrm{BSE}, E_{\mathrm{g}}^{\mathrm{G}_{0} \mathrm{~W}_{0-B S E}}$ calculations. The difference between the quasiparticle gap of a material and its optical gap is

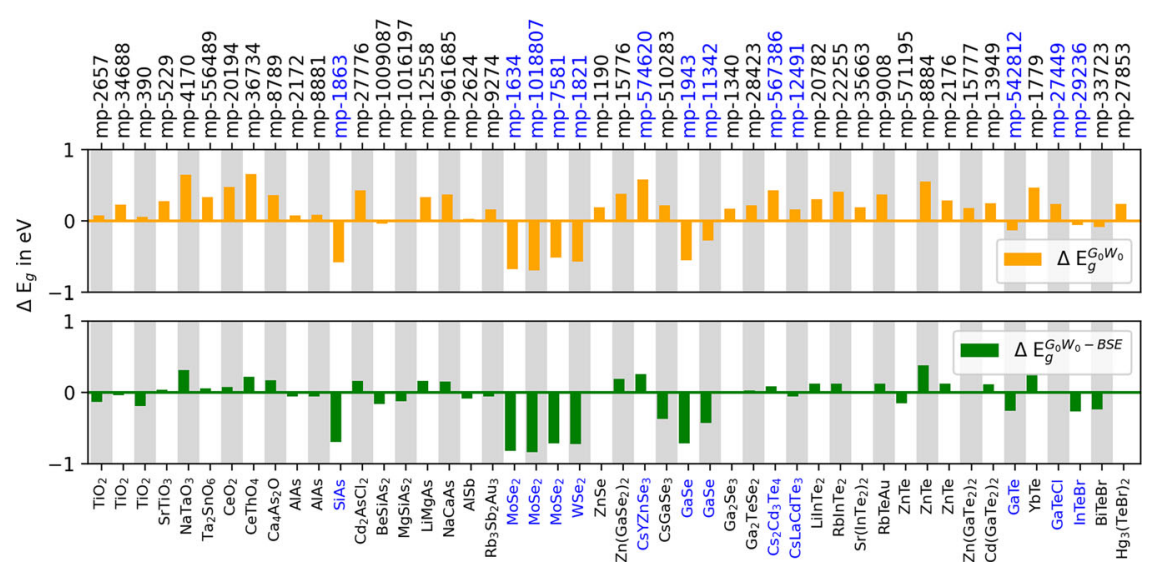

Fig. 1 Differences between $E_{g}^{\mathrm{HSE}}$ and $E_{\mathrm{g}}^{\mathrm{G}_{0} \mathrm{~W}_{0}}$ (orange) as well as $E_{\mathrm{g}}^{\mathrm{G}_{0} \mathrm{~W}_{0-\mathrm{BSE}}}$ (green). The chemical formula and Materials Project id's (mp-id) for each material are shown on the bottom and top $x$-axis ticks respectively. The layered materials have been labeled in blue. 
Table 1. Comparison between the computed (Theo) and experimental (Expt) lattice constants $\left(a, b, c\right.$ in $\AA$, and $a, \beta, \gamma$ in ${ }^{\circ}$ ), the Materials Project material ids ( $\mathrm{mp}$-id) and spacegroup (SG) of all the layered materials considered in this study.

\begin{tabular}{|c|c|c|c|c|c|c|c|c|c|c|c|c|c|c|}
\hline $\mathrm{mp}$-id & Formula & SG & $a^{\text {Theo }}$ & $b^{\text {Theo }}$ & $c^{\text {Theo }}$ & $a^{\text {Theo }}$ & $\beta^{\text {Theo }}$ & $\gamma^{\text {Theo }}$ & $a^{\text {Expt }}$ & $b^{\text {Expt }}$ & $c^{\text {Expt }}$ & $a^{\text {Expt }}$ & $\beta^{\operatorname{Expt}}$ & $\gamma^{\text {Expt }}$ \\
\hline mp-1943 & GaSe & $\mathrm{P}_{3} / \mathrm{mmc}$ & 3.80 & 3.80 & 15.73 & 90.00 & 90.00 & 120.00 & 3.75 & 3.75 & 15.99 & 90.00 & 90.00 & 120.00 \\
\hline $\mathrm{mp}-542812$ & GaTe & $\mathrm{C} 2 / \mathrm{m}$ & 17.61 & 4.14 & 10.62 & 90.00 & 104.60 & 90.00 & 17.40 & 4.08 & 10.46 & 90.00 & 104.50 & 90.00 \\
\hline mp-1634 & $\mathrm{MoSe}_{2}$ & $\mathrm{P}_{3} / \mathrm{mmc}$ & 3.33 & 3.33 & 13.17 & 90.00 & 90.00 & 120.00 & 3.29 & 3.29 & 12.93 & 90.00 & 90.00 & 120.00 \\
\hline$m p-1821$ & $\mathrm{WSe}_{2}$ & $\mathrm{P}_{3} / \mathrm{mmc}$ & 3.31 & 3.31 & 13.04 & 90.00 & 90.00 & 120.00 & 3.28 & 3.28 & 12.96 & 90.00 & 90.00 & 120.00 \\
\hline$m p-27449$ & $\mathrm{GaTeCl}$ & Pnnm & 5.90 & 14.59 & 4.13 & 90.00 & 90.00 & 90.00 & 5.85 & 14.47 & 4.08 & 90.00 & 90.00 & 90.00 \\
\hline mp-29236 & InTeBr & $\mathrm{P} 2_{1} / \mathrm{c}$ & 7.46 & 7.70 & 8.32 & 90.00 & 115.45 & 90.00 & 7.35 & 7.58 & 8.34 & 90.00 & 117.61 & 90.00 \\
\hline mp-569454 & $\mathrm{CdHgAsBr}$ & Pmma & 5.17 & 8.93 & 9.80 & 90.00 & 90.00 & 90.00 & 4.70 & 8.79 & 9.78 & 90.00 & 90.00 & 90.00 \\
\hline$m p-574620$ & $\mathrm{CsYZnSe}_{3}$ & $\mathrm{Cmcm}$ & 4.14 & 15.82 & 10.93 & 90.00 & 90.00 & 90.00 & 4.14 & 15.81 & 10.93 & 90.00 & 90.00 & 90.00 \\
\hline
\end{tabular}

precisely its EBE. One should note that, $\mathrm{G}_{0} \mathrm{~W}_{0}$ refers to non-selfconsistent GW approximation. Here, the single-particle Green's function, $G_{0}$, and the screened Coulomb interaction, $W_{0}$, are calculated using Kohn-Sham eigenvalues and eigenfunctions ${ }^{38}$.

We have calculated the root mean square difference (RMSD), defined as ${\sqrt{\frac{1}{N} \sum_{i=1}^{N}\left(\Delta E_{\mathrm{g}, \mathrm{i}}\right)}}^{2}$, for the gaps obtained from $\mathrm{G}_{0} \mathrm{~W}_{0}$ and $\mathrm{G}_{0} \mathrm{~W}_{0}$-BSE with the $E_{\mathrm{g}}^{\mathrm{HSE}}$ values for all the 48 materials. Where $E_{\mathrm{g}, \mathrm{i}}$ is the bandgap of the $i^{\text {th }}$ of the $N=48$ materials considered in this study. Note that a positive $\Delta E_{\mathrm{g}, \mathrm{i}}$ signifies underestimated $E_{\mathrm{g}}^{\mathrm{HSE}}$. From Fig. 1 it is evident that except the layered materials, the $E_{\mathrm{g}}^{\mathrm{HSE}}$ is almost always significantly underestimated compared to the QP gap obtained from $\mathrm{G}_{0} \mathrm{~W}_{0}$ calculations. We find an RMSD value of $0.16 \mathrm{eV}$ for optical gaps and $0.31 \mathrm{eV}$ for QP gaps for the nonlayered materials considered in this study, which highlights the importance of studying photocatalytic materials with the GW-BSE methodology. In the case of layered materials, the difference between previously reported $E_{\mathrm{g}}^{\mathrm{HSE}}$ and $E_{\mathrm{g}}^{\mathrm{G}_{0} \mathrm{~W}_{0-B S E}}$ resulting from our calculation, has a different origin, which we will discuss in the following section.

\section{Layered materials and Van der Waals correction}

We observe a large difference in the previously reported $E_{\mathrm{g}}^{\mathrm{HSE}}$ values and our $E_{\mathrm{g}}^{\mathrm{G}_{0} W_{0}}$ as well as $E_{\mathrm{g}}^{\mathrm{G}_{0} \mathrm{~W}_{0-B S E}}$ values for the layered materials. This difference originates primarily from the change in the lattice parameters due to the Van der Waals (vdW) corrections included in our work. Singh et al. used the lattice parameters computed by the Materials Project for their computational screening of more than 68,000 potential candidates ${ }^{18}$. The Materials Project structural relaxation simulations do not account for any vdW corrections. In contrast, in this work we have included vdW corrections using the non-local vdW density functional (vdW-DF) proposed by Dion et al. ${ }^{39}$ with optimized exchange functionals (optB88) ${ }^{40}$. Once we obtain an accurate lattice structure we have used the traditional PBE functional to obtain Kohn-Sham wavefunction and eigenvalues for the mean-field starting point for subsequent GW calculation.

In Table 1 we compare the lattice parameters obtained by using vdW corrections for the structural optimization in case of the layered materials with the experimental lattice parameters and we find a very good agreement. The experimental lattice constants are obtained from the ICSD ${ }^{41}$ lattice constants available through the Materials Project database. We also find that for these layered materials the Materials Project computed lattice parameters, and hence those used in Singh et al.'s work, are typically underestimated in the direction of the vdW bonding. This gives rise to larger $E_{\mathrm{g}}^{\mathrm{HSE}}$ bandgaps in Singh et al.'s work and the significantly smaller $E_{\mathrm{g}}^{\mathrm{G}_{0} \mathrm{~W}_{0}}$ and $E_{\mathrm{g}}^{\mathrm{G}_{0} \mathrm{~W}_{0-B S E}}$ gaps in this work. A RMSD of $0.54 \mathrm{eV}$ for optical gaps and $0.47 \mathrm{eV}$ for QP gaps is observed for the layered materials considered in this study, underscoring the importance of $\mathrm{vdW}$ corrections. See Supplementary Table 1 for the lattice parameters of all the 48 materials obtained from the vdW corrected functional.

In the case of the direct bandgap layered materials, GaSe and $\mathrm{GaTe}$, the optical gap is found to be smaller than $1.7 \mathrm{eV}$, the lower end the of desirable 1.7-3.5 eV bandgap window of a visible-light photoabsober ${ }^{18}$. For the dichalcogenides $\mathrm{MoSe}_{2}$ (all three phases) and $\mathrm{WSe}_{2}$ the direct absorption edge is just above $1.5 \mathrm{eV}$, however, at finite temperature the phonon assisted indirect absorption edge will be at an energy lower than $1.5 \mathrm{eV}$. These observations suggest that these materials may not be ideal for visible-light photocatalysis due to their low optical gap.

\section{Accuracy of $\mathbf{G W}_{\mathbf{0}}$ in comparison to $\mathbf{G}_{\mathbf{0}} \mathbf{W}_{\mathbf{0}}$}

We also computed the bandgaps using the partially self-consistent $\mathrm{qpGW}_{0}$ and $\mathrm{qpGW}_{0}-\mathrm{BSE}$ calculations. For brevity we use $\mathrm{GW}_{0}$ and $\mathrm{GW}_{0}-\mathrm{BSE}$ instead of $\mathrm{qpGW}_{0}$ and $\mathrm{qpGW}_{0}-\mathrm{BSE}$, respectively, in the rest of the article. In $\mathrm{GW}_{0}$, the eigenfunctions from ground-state calculations (i.e. DFT) are maintained, and $\mathrm{G}_{0} \mathrm{~W}_{0}$ calculations are performed to compute QP energies, which is then updated in the calculation of the Green's function, $G^{31}$. These calculations show a significant increase in the QP gap for most of the oxides as well as several other materials such as $\mathrm{CsYZnSe}_{3}$ and $\mathrm{RbTeAu}$ (see Supplementary Table 2). As a result, the RMSD value for the QP gap obtained from the $\mathrm{GW}_{0}$ calculation shoots up to $0.8 \mathrm{eV}$. A relatively low RMSD value of $0.32 \mathrm{eV}$ is obtained for the optical gap, as the EBE's are high ( 0.5 eV) for these materials.

Table 2 presents a comparison of the $E_{\mathrm{g}}^{\mathrm{G}_{0} \mathrm{~W}_{0-B S E}}$ and $E_{\mathrm{g}}^{\mathrm{GW}} \mathrm{W}_{0-\mathrm{BSE}}$ with experimentally measured optical gaps. Experimental measurements of the optical gaps were available in the literature for 10 of 
Table 2. Comparison between optical gaps obtained from $\mathrm{G}_{0} \mathrm{~W}_{0}-\mathrm{BSE}$,

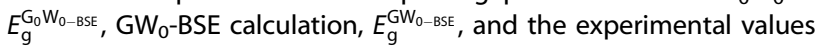
previously reported in the literature.

\begin{tabular}{llll}
\hline Material & $E_{\mathrm{g}}^{\mathrm{G}_{0} \mathrm{~W}_{0-B S E}}$ & $E_{\mathrm{g}}^{\mathrm{GW}}$ & Experimental \\
\hline $\mathrm{TiO}_{2}$ (rutile) & 3.07 & 3.91 & $3.03^{85}$ \\
$\mathrm{TiO}_{2}$ (anatase) & 3.31 & 4.19 & $3.42^{86}$ \\
$\mathrm{SrTiO}_{3}$ & 3.24 & 4.21 & $3.30^{87}$ \\
$\mathrm{CeO}_{2}$ & 3.68 & 6.04 & $3.78^{88}$ \\
$\mathrm{MoSe}_{2}$ & 1.08 & 1.14 & $1.09^{89}$ \\
$\mathrm{WSe}_{2}$ & 1.18 & 1.25 & $1.20^{89}$ \\
$\mathrm{ZnSe}$ & 2.30 & 2.65 & $2.82^{90}$ \\
$\mathrm{ZnTe}$ & 2.22 & 2.49 & $2.39^{90}$ \\
$\mathrm{AlAs}$ & 2.04 & 2.26 & $2.15^{91}$ \\
AlSb & 1.71 & 1.89 & $1.62^{91}$ \\
\hline All values & & &
\end{tabular}

All values are in eV. The $E_{\mathrm{g}}^{\mathrm{G}_{0} \mathrm{~W}_{0-\text {-BSE }}}$ is in better agreement with the experimentally measured values in comparison to the $E_{\mathrm{g}}^{\mathrm{GW}}$-BSE .

the materials that we considered in this work-four oxides, three selenides, one arsenide, and an antimonide. The $E_{\mathrm{g}}^{\mathrm{G}_{0} \mathrm{~W}_{0-B S E}}$ has a better agreement $(\mathrm{RMSD}=0.19 \mathrm{eV})$ with the experimentally obtained optical gaps than the $E_{\mathrm{g}}^{\mathrm{GW}}$-BSE $(\mathrm{RMSD}=0.86 \mathrm{eV})$. This might be due to the fact that the four oxides are wide bandgap materials and self-consistency tends to overestimate the bandgap in such materials ${ }^{42}$. The only materials for which the $\mathrm{GW}_{0}-\mathrm{BSE}$ provides better agreement with experiments is $\mathrm{ZnSe}$ and $\mathrm{ZnTe}$. In case of $\mathrm{MoSe}_{2}$ and WSe $\mathrm{W}_{2}$ self-consistency does not change the QP gap significantly and both $\mathrm{G}_{0} \mathrm{~W}_{0}-\mathrm{BSE}$ and $\mathrm{GW}_{0}-\mathrm{BSE}$ provide excellent agreement with experiments. Therefore, in all the subsequent calculations reported in this study we have restricted ourselves with $\mathrm{G}_{0} \mathrm{~W}_{0}$ level of calculation to find the QP energies.

\section{Design of the screening criteria}

We designed three screening criteria to identify the photocatalysts with the most suitable optical properties among the 48 materials considered in this study. Below we describe these criteria in detail.

As we have already discussed, high EBE is not desirable for photocatalytic or photovoltaic applications. Therefore, we consider materials that have an EBE of less than $200 \mathrm{meV}$ as suitable candidates for photocatalysis. The motivation behind choosing $200 \mathrm{meV}$ as the EBE criteria limit came from looking at the experimental EBE of materials that are currently accepted as a viable choice for photocatalytic applications, such as the anatase phase of $\mathrm{TiO}_{2}(\mathrm{EBE}=180 \mathrm{meV})^{43}$ and $\mathrm{MoS}_{2}(\mathrm{EBE}=240 \mathrm{meV})^{44}$. The cut off of $200 \mathrm{meV}$ was chosen because we wanted to look for materials that are as good as (if not better than) the most promising photocatalysts known in the literature (e.g. $\left.\mathrm{TiO}_{2}\right)$. We find that 26 of the 48 materials considered in this study have an EBE of less than $200 \mathrm{meV}$. We examine these materials in detail in the Outcome of Screening for Optical Properties section.

While it is necessary for a photoabsorber or photocatalytic material to have an optical gap in the visible-light region to absorb a significant amount of sunlight, it is not sufficient. It is also necessary that the material is able to absorb visible light of all possible polarizations. The amount of incident radiation of a certain frequency and polarization that will be absorbed by a material is determined by its absorption coefficient at that particular frequency and polarization.

Considering this, we propose to use an additional screening criteria to search for good photoabsorber/photocatalytic materials, one that is based on their integrated absorption coefficient, $a_{\text {int, }}$ for light polarization along the $x(\| \hat{a}), y(\| \hat{b})$ and $z(\| \hat{c})$ crystal axes, namely $a_{\mathrm{int}}^{x}, a_{\mathrm{int}}^{y}$, and $a_{\mathrm{int}}^{z}$.

$a_{\text {int }}$ is computed by integrating the frequency dependent absorption coefficient $(a(\omega))$ in the $1.7-3.5 \mathrm{eV}$ range ${ }^{18}$ for polarization of light along $x(\| \hat{a}), y(\| \hat{b})$, and $z(\| \hat{c})$ axes. To compute $a(\omega)$ one needs both the real $\left(\epsilon_{1}(\omega)\right)$ and the imaginary $\left(\epsilon_{2}(\omega)\right)$ part of the dielectric function. In case of light polarization along $x_{\text {, }}$

$$
a_{\mathrm{int}}^{x}=\int_{\omega_{\min }}^{\omega_{\max }} \alpha^{x}(\omega) d \omega \text { where } a^{x}(\omega)=\frac{2 \pi\left(\left|\sqrt{\left(\epsilon_{1}^{x}(\omega)\right)^{2}+\left(\epsilon_{2}^{x}(\omega)\right)^{2}}\right|-\epsilon_{1}^{x}(\omega)\right)^{1 / 2}}{\lambda}
$$

where $\omega$ and $\lambda$ are the frequency and wavelength of incident radiation. Here, we obtain $\epsilon_{2}(\omega)$ directly by solving BSE and use the Kramers-Kronig relation to calculate $\epsilon_{1}(\omega)$.

Through this integration of the $a(\omega)$ in the energy range of visible photons and averaging for different light polarization, one can eliminate all the materials that have excitons in the desired energy range but are optically inactive or 'dark'. Optically 'dark' excitons exist as a solution to the BSE but have zero oscillator strength, hence, do not contribute to optical absorption ${ }^{45,46}$.

To find a suitable cutoff value of $a_{\mathrm{int}}^{\mathrm{avg}}$ (defined as $\frac{a_{\mathrm{int}}^{x}+a_{\mathrm{int}}^{y}+a_{\mathrm{int}}^{z}}{3}$ ) for identifying promising photocatalysts we again look at the most widely studied material for this application, the anatase phase of $\mathrm{TiO}_{2}$. However, the anatase $\mathrm{TiO}_{2}$ is not used as a visible-light photocatalyst. The optimum performance for this material can only be achieved under exposure to UV radiation. Therefore, we have computed the $a_{\text {int }}^{\text {avg }}$ in the range of $3.5-4.2 \mathrm{eV}$ for $\mathrm{TiO}_{2}$ and used that as the cutoff. We find the value of $a_{\text {int }}^{\text {avg }}$ for $\mathrm{TiO}_{2}$ (in the UV range) is $3.6 \times 10^{4} \mathrm{~cm}^{-1} \mathrm{eV}$ which is almost three times smaller than the $a_{\text {int }}^{\text {avg }}$ for silicon $\left(10.5 \times 10^{4} \mathrm{~cm}^{-1} \mathrm{eV}\right.$ in the visible range), the most widely used photoabsorber of this time. The $a_{\text {int }}^{\text {avg }}$ for silicon was computed using experimental data for $a(\omega)^{47}$ in the 1.7-3.5 eV range.

Anisotropic absorption of light is undesirable as it would make certain surfaces of a poly-crystalline sample less capable of absorbing incident unpolarized solar radiation, and hence, reducing the efficiency of the solar-to-chemical energy conversion process. Thus, we consider an additional screening criteria, $a_{\mathrm{int}}^{\text {aniso, }}$, that assess the anisotropy in the integrated absorption coefficients along $x, y$, and $z$ polarization axis. Here, $a_{\mathrm{int}}^{\text {aniso }}=\frac{\operatorname{minimum}\left(a_{\mathrm{int}}^{\mathrm{x}}, a_{\mathrm{int}}^{y}, a_{\mathrm{int}}^{z}\right)}{\operatorname{maximum}\left(a_{\mathrm{int}}^{\mathrm{x}}, a_{\mathrm{int}}^{\mathrm{v}}, a_{\mathrm{int}}^{2}\right)}$. By computing $a_{\text {int }}^{\text {aniso }}$ one can eliminate materials that absorb lights with preferential polarization. We chose an $a_{\text {int }}^{\text {aniso }}$ of $>0.8$ as the criteria of selection for this study.

In summary, the three criteria that we propose for screening photocatalysts with suitable optical properties are,

(1) $\mathrm{EBE}<200 \mathrm{meV}$

(2) $a_{\text {int }}^{\text {avg }}>3.6 \times 10^{4} \mathrm{~cm}^{-1} \mathrm{eV}$

(3) $a_{\text {int }}^{\text {aniso }}>0.8$

\section{Outcome of screening for optical properties}

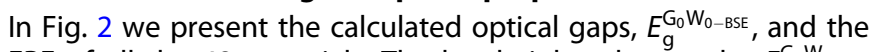
EBE of all the 48 materials. The bar heights denote the $E_{\mathrm{g}}^{\mathrm{G}_{0} \mathrm{~W}_{0-B S E}}$ and their colors denote the EBE. Most of the oxides considered here have high EBE's, making them unappealing for photovoltaic and photocatalytic applications. We, however, find 26 materials with very low EBE's of $<200 \mathrm{meV}$. The EBEs of all the 48 materials computed from both $\mathrm{G}_{0} \mathrm{~W}_{0}$-BSE as well as $\mathrm{GW}_{0}-\mathrm{BSE}$ are listed in the Supplementary Table 2.

With the exception of $\mathrm{NaCaAs}$, all the materials considered in

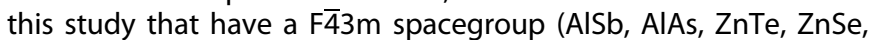
and LiMgAs) have EBE $<200 \mathrm{meV}$. All of these materials have a Zinc-blend crystal structure except LiMgAs, which has an additional $\mathrm{Mg}$ atom at the center of the unit cell. AlAs and ZnTe 


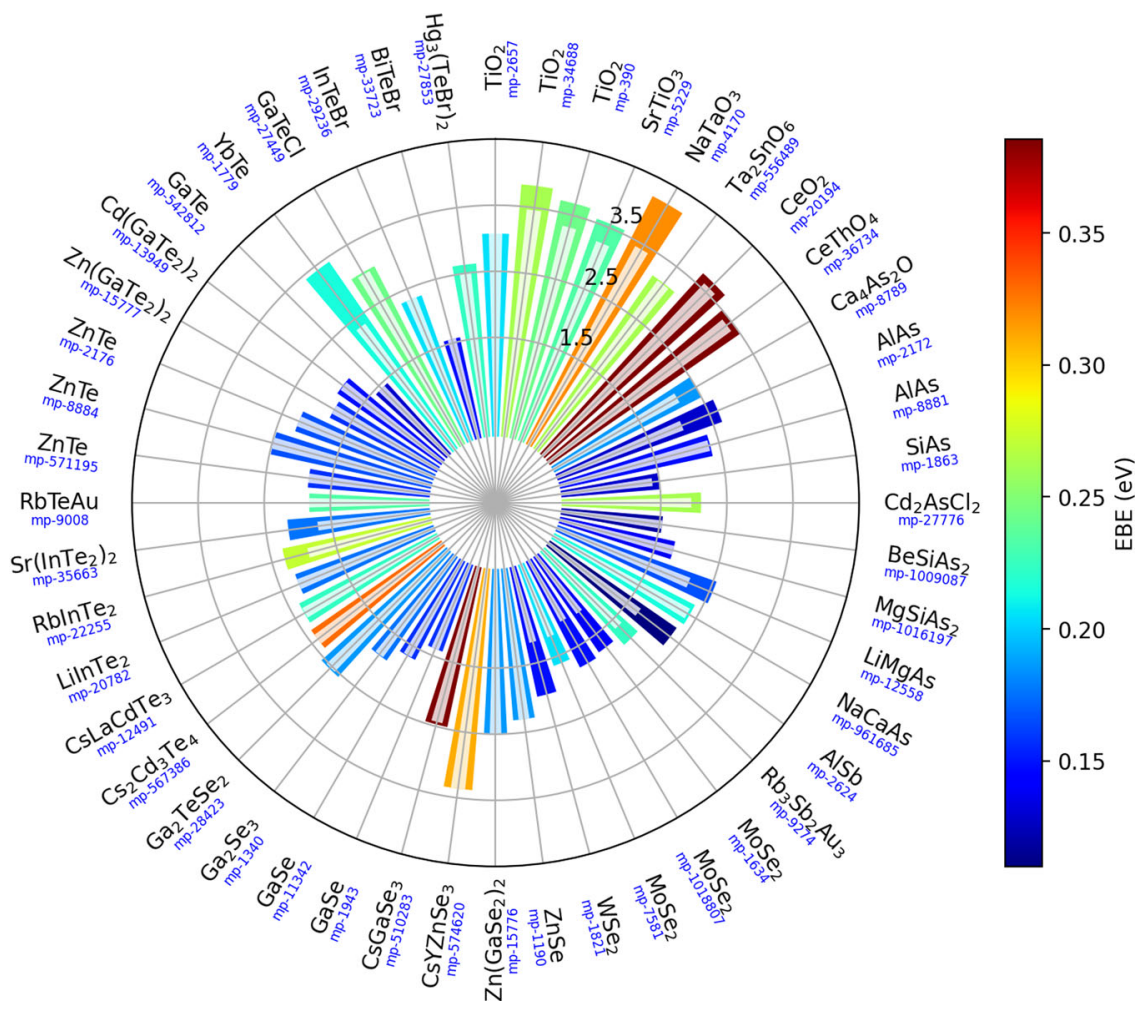

Fig. 2 The bar heights denote the optical gap, $E_{\mathrm{g}}^{\mathrm{G}_{0} \mathrm{~W}_{0-B E}}$ in $\mathrm{eV}$, and their colors denote the exciton binding energy (EBE) as obtained from $\mathbf{G}_{0} \mathbf{W}_{\mathbf{0}}$-BSE calculations for the $\mathbf{4 8}$ materials. We find that $\mathbf{2 6}$ of the $\mathbf{4 8}$ materials have EBE less than $0.2 \mathrm{eV}$. The radial heights corresponding to $1.5,2.5$, and $3.5 \mathrm{eV}$ optical gaps are labeled in the figure. The indirect absorption edges are shown as white bars for each material. For direct gap materials, they are equal to the optical gap.

with Wurtzite crystal structure (spacegroup $\mathrm{P}_{3} \mathrm{mc}$ ) also have quite low EBE's, 145 and 169 meV respectively. Among the materials with low EBE $(<150 \mathrm{meV})$ we find two group-II-IV-V 2 ternary compounds with body centered tetragonal chalcopyrite structure (spacegroup $1 \overline{4} 2 \mathrm{~d}$ ) namely, $\mathrm{MgSiAs}_{2}$ and $\mathrm{BeSiAs}_{2}$. These two materials are known to be promising materials for spintronics, electronic and optoelectronic applications such as infrarednonlinear optical material, solar energy converters, infrared detectors, visible and invisible light-emitting diodes ${ }^{48,49}$.

A group of telluride materials $\left(\mathrm{Zn}\left(\mathrm{GaTe}_{2}\right)_{2}, \mathrm{Cd}\left(\mathrm{GaTe}_{2}\right)_{2}\right.$, and $\mathrm{Sr}$ $\left.(\operatorname{lnTe})_{2}\right)$, which are generally synthesized by preparing solid solutions of $\mathrm{A}_{2} \mathrm{Te}_{3}\left(\mathrm{~A}=\mathrm{Ga}\right.$, In) with other $\mathrm{A}^{\text {II }} \mathrm{B}^{\text {IV }}$ tellurides ${ }^{50}$, also exhibit low EBE's (140-180 meV). The layered chalcogenides (GaTe, GaSe, $\mathrm{MoSe}_{2}$, and $\mathrm{WSe}{ }_{2}$ ) have $\mathrm{EBE}<200 \mathrm{meV}$ as well. Interestingly, the different phases of these chalcogenides do not have significantly different EBE. Most of the layered dichalcogenides $\left(\mathrm{MoSe}_{2}, \mathrm{WSe}_{2}\right)$ with hexagonal crystal unit cell (spacegroup $\mathrm{P}_{3} / \mathrm{mmc}$ ) have EBE in the range of $(130-150 \mathrm{meV}) . \mathrm{MoSe}_{2}$ in a hypothetical phase with rhombohedral unit cell (spacegroup $\mathrm{R} 3 \mathrm{~m}$ ) has a slightly larger EBE of $208 \mathrm{meV}$.

In three-dimensional (3D) systems such as bulk semiconductors, the Coulomb interaction between an electron and a hole is effectively screened $(\epsilon \sim 10)$ by other electrons and therefore results in a weakly bound exciton (EBE $\sim 0.1 \mathrm{eV})$. However, in lowdimensional materials or $2 \mathrm{D}$ materials the electronic screening is less effective as the e- $h$ pair can interact partially through vacuum. Consequently, 2D materials show more pronounced excitonic effects and EBEs order of magnitude larger than their bulk counterpart ${ }^{51,52}$. State-of-the-earth first principles GW-BSE calculations have been employed successfully in exploring excitonic effects in $2 \mathrm{D}$ materials such as hexagonal $\mathrm{BN}$ sheet ${ }^{51}$, graphane ${ }^{53}$, monolayered $\mathrm{MoS}_{2}{ }^{25}$, and semi metallic graphene ${ }^{54}$. In our study, we have examined the bulk layered form of the $2 \mathrm{D}$ materials. But one can expect a much higher EBE for the 2D forms of these materials.

Figure 3 shows the $a_{\text {int }}$ computed for the case of light polarization along $x$ (red bars), $y$ (blue bars), and $z$ (green bars) axis for all 48 materials under consideration. We find that among materials with low EBE $(<200 \mathrm{meV}) 14$ materials have $a_{\mathrm{int}}^{\text {avg }}$ values larger than the cutoff of $3.6 \times 10^{4} \mathrm{~cm}^{-1} \mathrm{eV}$.

In Fig. 4 we show the absorption spectra of four representative materials, BeSiAs 2 (mp-1009087), GaSe (mp-11342), BiTeBr (mp33723) and AISb (mp-2634). The excitation energies for each of these materials are shown as vertical lines in the yellow panels underneath their spectra.

First, let us examine the case of $\mathrm{BeSiAs}_{2}$ (Fig. 4a). For this material, we can see the lowest energy exciton is at $1.54 \mathrm{eV}$, its optical gap, and there are quite a few excitons in the $2-2.5 \mathrm{eV}$ energy range. However, most of these excitons are 'dark', i.e. they do not yield an appreciable $a_{\text {int }}$. Most of the 'bright' excitons for BeSiAs $_{2}$ lie above $\sim 3 \mathrm{eV}$ and thus this material has a low $a_{\text {int }}$ value in the visible-light region. Hence it does not meet the cutoff for criteria 2.

In the case of GaSe, which has a $1.67 \mathrm{eV}$ optical gap, there are very few bright excitons below $2 \mathrm{eV}$ and some bright excitons above $2.7 \mathrm{eV}$, see Fig. 4b. The low number of excitons in the visible frequency range results in a poor $a_{\text {int }}$ value. Thus, this material also does not meet the criteria 2 cutoff.

Transition metal dichalcogenides $\mathrm{MoSe}_{2}$ and $\mathrm{WSe}_{2}$ and other layered material such as BiTeBr (Fig. 4c) absorb significantly poorly if the light polarization is along the $z$-axis ( $30 \%$ compared to light polarization along $x$ or $y$ ). This results in a low $a_{\mathrm{int}}^{\text {aniso }}$ for many of the layered materials. For example, $\mathrm{GaTeCl}$ and $\mathrm{CsYZnSe}_{3}$ which are also layered materials show significantly lower absorption if the light polarization is along the axis with the vdW bonding. In the case of $\mathrm{GaTeCl}\left(\mathrm{CsYZnSe}_{3}\right)$ we see 66 (79) \% and 92 (38) \% 


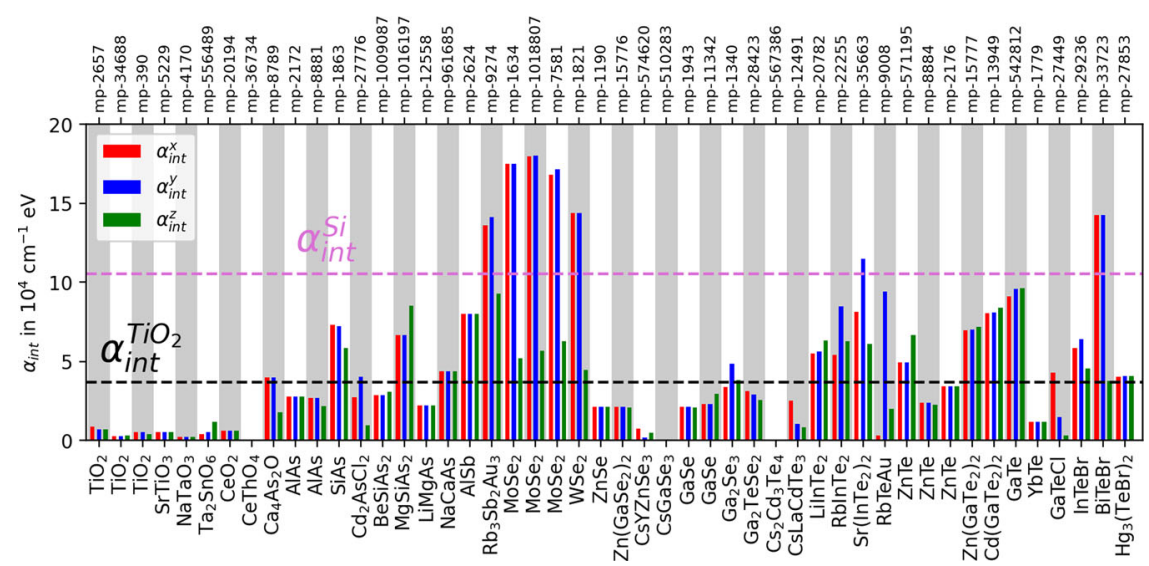

Fig. 3 Integrated absorption coefficient $\left(a_{\text {int }}\right)$ in case of light polarization along $x$ (red bars), $y$ (blue bars), and $z$ (green bars) axis for all the materials under consideration. We have integrated the absorption coefficient over the 1.7-3.5 eV energy range to show the degree of anisotropy in absorption for the visible region of the solar spectrum. Horizontal dashed lines denote the $a_{\text {int }}$ values for silicon (magenta) and $\mathrm{TiO}_{2}$ (black).
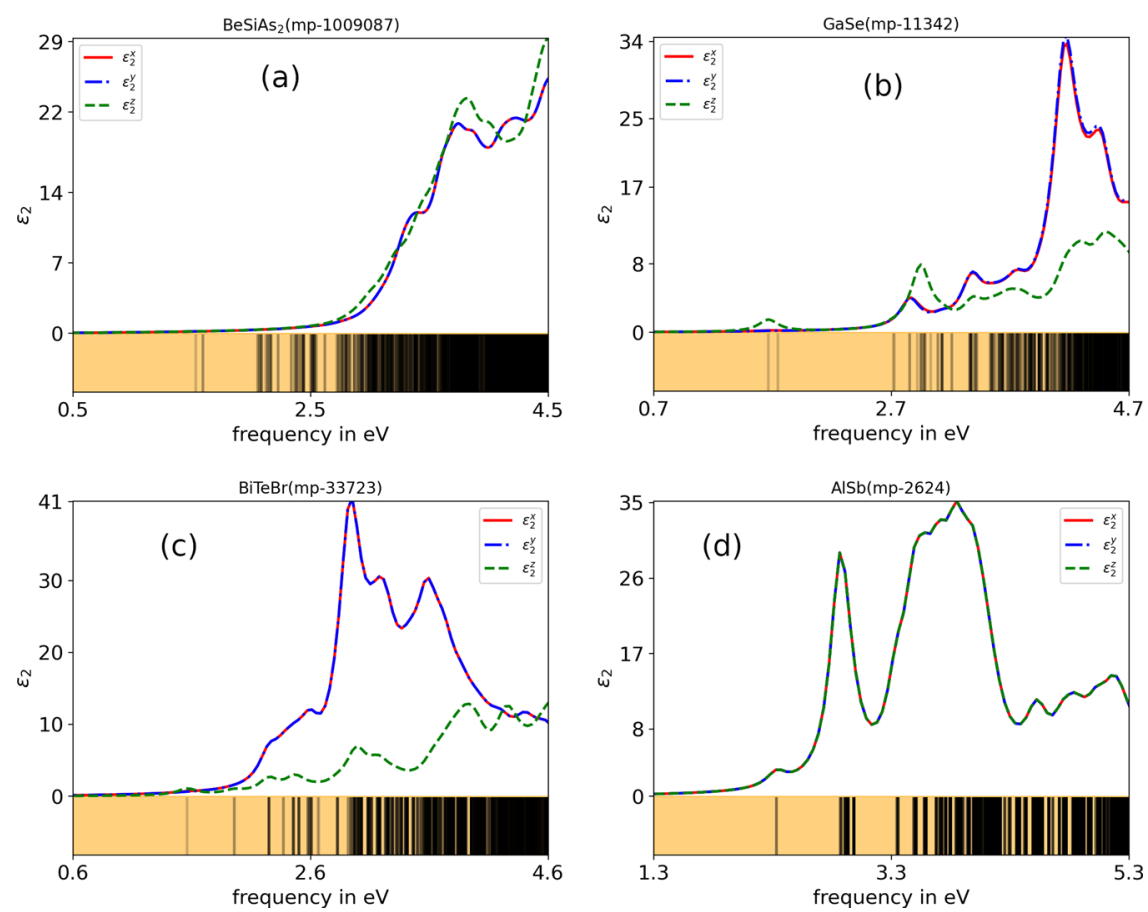

Fig. 4 The imaginary part of the dielectric function, $\varepsilon_{2}(\omega)$, for light polarization along $x$ (red), $y$ (blue), and $z$ (green) axis obtained from $\mathbf{G}_{\mathbf{0}} \mathbf{W}_{\mathbf{0}}$-BSE calculation for four selected materials. a BeSiAs (mp-1009087), b GaSe (mp-11342), c BiTeBr (mp-33723), and d AlSb (mp-2624). The yellow panel on the bottom of each figure shows the excitation energies with vertical lines.

decrease in total visible-light absorption if the light polarization is along the $y$ and $z$-axis respectively compared to if the polarization is along the $x$-axis. $\mathrm{Ga}_{2} \mathrm{Se}_{3}, \mathrm{RblnTe}_{2}, \mathrm{Sr}(\operatorname{InTe})_{2}, \mathrm{ZnTe} \mathrm{Ta}_{2} \mathrm{SnO}_{6}$ are some of the other materials which have a preference in absorbing visible lights of certain polarization than the others.

The absorption spectra of one of the gifted materials AISb (mp2634), which satisfies both the criteria 2 and 3 has been shown in Fig. $4 d$. It has an EBE of $110 \mathrm{meV}, a_{\mathrm{int}}^{\text {avg }}$ of $7 \times 10^{4} \mathrm{~cm}^{-1} \mathrm{eV}$, and $a_{\text {int }}^{\text {aniso }}$ of 1 (cubic system).

The absorption spectra of all the 48 materials can be found in the Supplementary Fig. 3. The Supplementary Table 3 lists the $a_{\text {int }}^{\text {aniso }}, a_{\mathrm{int}}^{\text {avg }}, a_{\mathrm{int}}^{\mathrm{x}}, a_{\mathrm{int}}^{\mathrm{y}}$, and $a_{\mathrm{int}}^{z}$ in the visible-light (1.7-3.5 eV) range of all the 48 materials.

In Fig. 5 we show a color-coded map to indicate which of the screening criteria are satisfied by each of these 48 materials.
Materials that satisfy the EBE criteria are shaded in blue. Materials that satisfy both criteria 2 and 3 of meeting the cutoff of $a_{\text {int }}^{\text {avg }}$ and $a_{\text {int }}^{\text {aniso }}$ in the visible-light region (1.7-3.5 eV) are shaded in orange. Materials that satisfy criteria 2 and 3 in the UV region (3.5-4.2 eV) are shaded in green. In this color-coded map, we can see that only six materials meet the three criteria for visible-light photocatalysis. These six materials are AlSb, SiAs, GaTe, $\mathrm{Zn}\left(\mathrm{GaTe}_{2}\right)_{2}, \mathrm{Cd}\left(\mathrm{GaTe}_{2}\right)_{2}$, and $\mathrm{LilnTe}_{2}$. All of these materials have been previously synthesized ${ }^{18}$. SiAs and GaTe are layered materials. These layered materials can be extracted in two-dimensional forms, which are known to offer high specific surface area, tunability, and potential for heterostructuring ${ }^{55-57}$. In a recent computational study, Torrissi et al. have shown the two-dimensional SiAs is selective for CO production and has good absorption in the visible-light region ${ }^{55}$. On the other hand, two-dimensional GaTe has been shown to 


\begin{tabular}{|c|c|c|c|c|c|c|c|}
\hline $\begin{array}{c}\mathrm{TiO}_{2} \\
\mathrm{mp}-2657\end{array}$ & $\begin{array}{c}\mathrm{TiO}_{2} \\
\mathrm{mp}-34688\end{array}$ & $\begin{array}{c}\mathrm{TiO}_{2} \\
\mathrm{mp}-390\end{array}$ & $\begin{array}{c}\mathrm{SrTiO}_{3} \\
\mathrm{mp}-5229\end{array}$ & $\begin{array}{c}\mathrm{NaTaO}_{3} \\
\mathrm{mp}-4170\end{array}$ & $\begin{array}{c}\mathrm{Ta}_{2} \mathrm{SnO}_{6} \\
\mathrm{mp}-556489\end{array}$ & $\begin{array}{c}\mathrm{CeO}_{2} \\
\mathrm{mp}-20194\end{array}$ & $\begin{array}{c}\mathrm{CeThO}_{4} \\
\mathrm{mp}-36734\end{array}$ \\
\hline $\begin{array}{l}\mathrm{Ca}_{4} \mathrm{As}_{2} \mathrm{O} \\
\mathrm{mp}-8789\end{array}$ & $\begin{array}{c}\text { AlAs } \\
\text { mp-2172 }\end{array}$ & $\begin{array}{c}\text { AlAs } \\
\text { mp-8881 }\end{array}$ & $\begin{array}{c}\text { SiAs } \\
\text { mp-1863 }\end{array}$ & $\begin{array}{c}\mathrm{Cd}_{2} \mathrm{AsCl}_{2} \\
\mathrm{mp}-27776\end{array}$ & $\begin{array}{c}\mathrm{BeSiAs}_{2} \\
\mathrm{mp}-1009087\end{array}$ & $\begin{array}{c}\mathrm{MgSiAs}_{2} \\
\mathrm{mp}-1016197\end{array}$ & $\begin{array}{l}\text { LiMgAs } \\
\text { mp-12558 }\end{array}$ \\
\hline $\begin{array}{c}\mathrm{NaCaAs} \\
\mathrm{mp}-961685\end{array}$ & $\begin{array}{c}\text { AlSb } \\
\mathrm{mp}-2624\end{array}$ & $\begin{array}{c}\mathrm{Rb}_{3} \mathrm{Sb}_{2} \mathrm{Au}_{3} \\
\mathrm{mp}-9274\end{array}$ & $\begin{array}{c}\mathrm{MoSe}_{2} \\
\mathrm{mp}-1634\end{array}$ & $\begin{array}{c}\mathrm{MoSe}_{2} \\
\mathrm{mp}-1018807\end{array}$ & $\begin{array}{c}\mathrm{MoSe}_{2} \\
\mathrm{mp}-7581\end{array}$ & $\begin{array}{c}\text { WSe }_{2} \\
\text { mp-1821 }\end{array}$ & $\begin{array}{c}\text { ZnSe } \\
\text { mp-1190 }\end{array}$ \\
\hline $\begin{array}{c}\mathrm{Zn}\left(\mathrm{GaSe}_{2}\right)_{2} \\
\mathrm{mp}-15776\end{array}$ & $\begin{array}{c}\mathrm{CsYZnSe}_{3} \\
\mathrm{mp}-574620\end{array}$ & $\begin{array}{c}\mathrm{CsGaSe}_{3} \\
\mathrm{mp}-510283\end{array}$ & $\begin{array}{c}\text { GaSe } \\
\text { mp-1943 }\end{array}$ & $\begin{array}{c}\text { GaSe } \\
\text { mp-11342 }\end{array}$ & $\begin{array}{c}\mathrm{Ga}_{2} \mathrm{Se}_{3} \\
\mathrm{mp}-1340\end{array}$ & $\begin{array}{c}\mathrm{Ga}_{2} \mathrm{TeSe}_{2} \\
\mathrm{mp}-28423\end{array}$ & $\begin{array}{c}\mathrm{Cs}_{2} \mathrm{Cd}_{3} \mathrm{Te}_{4} \\
\mathrm{mp}-567386\end{array}$ \\
\hline $\begin{array}{l}\mathrm{CsLaCdTe}_{3} \\
\mathrm{mp}-12491\end{array}$ & $\begin{array}{c}\text { LilnTe }_{2} \\
\text { mp-20782 }\end{array}$ & $\begin{array}{c}\mathrm{RbInTe}_{2} \\
\mathrm{mp}-22255\end{array}$ & $\begin{array}{l}\left.\mathrm{Sr}(\operatorname{InTe})_{2}\right)_{2} \\
\mathrm{mp}-35663\end{array}$ & $\begin{array}{l}\text { RbTeAu } \\
\text { mp-9008 }\end{array}$ & $\begin{array}{c}\text { ZnTe } \\
\mathrm{mp}-571195\end{array}$ & $\begin{array}{c}\text { ZnTe } \\
\text { mp-8884 }\end{array}$ & $\begin{array}{c}\mathrm{ZnTe} \\
\mathrm{mp}-2176\end{array}$ \\
\hline $\begin{array}{c}\mathrm{Zn}\left(\mathrm{GaTe}_{2}\right)_{2} \\
\mathrm{mp}-15777\end{array}$ & $\begin{array}{c}\mathrm{Cd}\left(\mathrm{GaTe}_{2}\right)_{2} \\
\mathrm{mp}-13949\end{array}$ & $\begin{array}{c}\text { GaTe } \\
\mathrm{mp}-542812\end{array}$ & $\begin{array}{c}\text { YbTe } \\
\text { mp-1779 }\end{array}$ & $\begin{array}{c}\mathrm{GaTeCl} \\
\mathrm{mp}-27449\end{array}$ & $\begin{array}{c}\text { InTeBr } \\
\mathrm{mp}-29236\end{array}$ & $\begin{array}{c}\text { BiTeBr } \\
\text { mp-33723 }\end{array}$ & $\begin{array}{l}\mathrm{Hg}_{3}(\mathrm{TeBr})_{2} \\
\mathrm{mp}-27853\end{array}$ \\
\hline
\end{tabular}

Fig. 5 The figure shows a color-coded map that indicates which of the screening criteria are satisfied by the $\mathbf{4 8}$ materials. The materials that satisfy the EBE criteria 1 are shaded in blue. The materials that satisfy both criteria 2 and 3 of meeting the cutoff of $a_{\text {int }}^{\text {avg }}$ and $a_{\text {int }}^{\text {aniso in the }}$ visible-light region (1.7-3.5 eV) are shaded in orange. The materials that satisfy criteria 2 and 3 in the UV region (3.5-4.2 eV) are shaded in green.

exhibit a sharp decrease in photoluminescence during the transition from the many to few-layer limit ${ }^{55}$.

The materials that satisfy all the criteria in the UV region are markedly more, total 18 . In Fig. 5 these materials are shaded in blue and green colors. We observe that most of the oxides do not make the list but several arsenides, tellurides, and selenides emerge as suitable candidates. Supplementary Table 4 lists the $a_{\mathrm{int}}^{\text {aniso }}, a_{\mathrm{int}}^{\text {avg }}, a_{\mathrm{int}}^{x}, a_{\mathrm{int}}^{y}$, and $a_{\mathrm{int}}^{z}$ in the UV-light range (3.5-4.2 eV) of all the 48 materials.

\section{Approximating EBE using Wannier-Mott Model}

In case of most inorganic semiconductors, excitons can be approximated as an electron in the conduction band that is bound with a hole in the valence band through some form of screened Coulomb interaction and can be described using the WM model ${ }^{58}$. This model uses effective mass approximation which describes electrons and holes as free particles that have a parabolic dispersion and are characterized by effective masses that depend on the crystal structure.

Within this approximation, the wavefunction of relative e-h motion or exciton can be found from the Schrodinger equation very similar to the one describing the electron state in a hydrogen atom where we replace the electron mass, $m_{0}$, with reduced effective mass, $\mu=\frac{m_{e} m_{h}}{m_{e}+m_{h}}$, and replace the unscreened Coulomb interaction, $\frac{e^{2}}{r}$, between the e-h pair with the screened one, $\frac{e^{2}}{c r}$. Where $m_{e}\left(m_{h}\right)$ is the electron (hole) effective masses and $\epsilon$ is the dielectric constant of the material. By solving the hydrogen atom like Schrodinger's equation we find the binding energy of the ground excited state $\left(\mathrm{EBE}_{\mathrm{Wm}}\right)$,

$$
\mathrm{EBE}_{\mathrm{WM}}=\frac{\mu e^{4}}{2 \hbar^{2} \epsilon^{2}}
$$

Computationally it is much less expensive to obtain EBE using the WM model than solving the BSE. The input parameters in WM model are the dielectric constant and the effective masses, which can be obtained with reasonable accuracy using less expensive DFT calculations ${ }^{59}$. Here, we test the applicability of WM model for the materials under consideration and the possibility of using WM instead of the expensive BSE calculations for future solar-materials discovery.

The WM approximation and the validity of Eq. 3 depends on the assumption that the interaction potential is slowly varying over the dimension of an unit cell or in other words the dimension of lowest energy exciton is much larger than the lattice dimension ${ }^{58}$. If we assume the validity of the WM model and use $\mu \sim m_{e}$ and $\epsilon \sim 10$ in Eq. 3, we get EBE $\sim 0.1 \mathrm{eV}$, which is of the same order of magnitude as the computed EBE of the materials we have considered in this study $(0.11-0.59 \mathrm{eV})$, which motivates us to test the validity of the WM model for these materials.

To find the degree of correlation between the EBE computed using BSE $\left(E_{B B E}\right)$ and WM model $\left(\left(E_{B B} E_{W M}\right)\right)$ we calculate the Pearson's correlation coefficient (PCC) and the Spearman's correlation coefficient $(\mathrm{SCC})^{60}$. PCC indicates the strength of linear relationship between two variables whereas, SCC does not assume any particular functional dependence to quantify the strength of the correlation. We find a PCC value of 0.85 and SCC value of 0.76 between the $E_{B E} E_{B S E}$ and the $E_{B E}$, which suggests a strong correlation between the two. The high PCC value also suggests that we can scale the EBE obtained from WM model by a scaling factor (3.04) (see Supplementary Table 5) to get a reasonable prediction for the values obtained by solving the BSE. We find that such a scaling of the $\mathrm{EBE}_{\mathrm{WM}}$ to obtain the $\mathrm{EBE}_{\mathrm{BSE}}$ results in a mean squared error of $0.11 \mathrm{eV}$ and root mean squared deviation of $0.12 \mathrm{eV}$, which is similar to the accuracy of standard GW-BSE calculation. Moreover, we have computed the confusion matrix between the $\mathrm{EBE}_{\mathrm{BSE}}$ and predicted (scaled) $\mathrm{EBE}$ from the WM model. To compute the confusion matrix we use $200 \mathrm{meV}$ as the cutoff to classify the materials as low (high) EBE materials. In the Supplementary Fig. 1 we have shown the (a) confusion matrix and, (b) the parity plot between the $\mathrm{EBE}_{\mathrm{BSE}}$ and predicted (scaled) EBE from the WM model. Based on the confusion matrix, we find that for 37 out of 48 materials ( $77 \%$ ) the WM model with the scaling predicts the correct classification. Therefore, it would be quite reasonable to use WM model for a computational data-driven discovery of materials that have low EBE instead of using the computationally much more expensive BSE calculations.

In case of the other two screening criteria based on the absorption coefficients used in this study, $a_{\text {int }}^{\text {avg }}$ and $a_{\text {aniso, one }}^{\text {avg }}$ requires the entire frequency dependent absorption spectra $(\epsilon(\omega)$ ). Unfortunately, most of the less expensive methods such as RPA don't take e-h interaction into account and therefore provide grossly inaccurate spectra and thus we can not develop a simple surrogate model for these quantities and have to solve the massively expensive BSE.

In summary, we have explored excitonic effects in the optical properties of 48 promising $\mathrm{CO}_{2}$ reduction photocatalysts. We have designed a screening strategy considering the optical properties of materials that are crucial for such photocatalysts, i.e, the exciton binding energies, the capability to absorb visible light, and the degree of anisotropy in the absorption of light with different polarizations. 
Using this screening strategy we have identified six materials that are suitable for visible-light photocatalysis. Interestingly, to our knowledge, none of these materials have been extensively studied to be used as photocatalysts for $\mathrm{CO}_{2}$ reduction. Three of these materials have been studied before, using various theoretical and experimental methodology, i.e, $\mathrm{SiAs}^{61,62}, \mathrm{AlSb}^{63,64}$, and $\mathrm{GaTe}^{65}$. However, the other three materials LilnTe $2, \mathrm{Zn}\left(\mathrm{GaTe}_{2}\right)_{2}$, and $\mathrm{Cd}\left(\mathrm{GaTe}_{2}\right)_{2}$ have remained largely unexplored. It is noteworthy that all of these materials have been previously experimentally synthesized ${ }^{64,66-70}$. Our screening strategy also identifies 18 materials that are suitable for UV-light photocatalysis.

GW-BSE calculations have been extremely successful in predicting excitonic and optical properties of materials with $s$ or $p$ electrons, however, a similar success has not been reproduced when applied to $d$-electron systems ${ }^{71}$. Nevertheless, the BSE has been proven to be reasonably successful in exploring strong excitonic effects observed in $\mathrm{Cu}_{2} \mathrm{O}^{72}, \mathrm{GaN}^{34}, \mathrm{YMnO}_{3}{ }^{73}$, rutile and anatase $\mathrm{TiO}_{2}{ }^{74}$. In case of TMOs, when compared to experimental absorption spectra calculated using GW-BSE, the position of excitonic peaks are found to be accurate within $\sim 0.2 \mathrm{eV}$. To make GW-BSE calculation more accurate when applied to TMOs, several methods have been proposed such as using more appropriate mean-field starting points such as DFT $+U$ or HSE, self-consistent GW calculation, going beyond GW approximation and using vertex correction. However, none of these solutions have been shown to be a universal method appropriate for all TMOs ${ }^{71}$. One should also note that, there exists significant technical difficulties that hamper a precise measurement of optical spectra for TMOs due to chemical defects (e.g. oxygen vacancies or presence of TM impurities) ${ }^{75}$. Therefore, our reported data using the state-of-the earth GW-BSE framework represents a first comprehensive map of the optical and excitonic properties of these materials and should serve as a reference for future calculations and experiments.

Using the relatively large amount of the high-quality EBE data from BSE calculations generated in this work, we show that the WM model can be used for estimating exciton binding energies in a computationally cheaper manner for large-scale computational material discovery of photoabsorber materials without the need of performing the extraordinarily expensive BSE calculations.

Overall, our work presents a paradigm towards the inclusion of optical properties for data-driven discovery of materials for solar energy conversion applications.

\section{METHODS}

\section{Ground-state calculations using DFT}

All the first-principles calculations reported in this study were performed using Vienna $\mathrm{Ab}$ initio Simulation Package $\mathrm{e}^{33,38,76}$. The Kohn-Sham wavefunctions and eigenvalues were obtained using a plane wave basis with an energy cutoff of $520 \mathrm{eV}$. We employ the non-local vdW-DF-optB88 exchange-correlation functional ${ }^{39,40}$ as it provides an excellent description of the vdWs interactions in layered materials. The Brillouin zone was sampled using a $k$-grid with 100 kpoints per $\AA^{-3}$ of the reciprocal cell. The crystal structures were obtained by relaxing the atomic positions and the lattice parameters by a energy convergence cutoff of $0.0005 \mathrm{eV} \times$ number of atoms in the cell, which has been found to produce well-converged structures in most instances ${ }^{77}$.

The macroscopic dielectric constants reported here were calculated using density functional perturbation theory following the formalism originally introduced by Baroni et al. ${ }^{78}$ and later implemented for projector-augmented wave methodology by Gajdoš et al..$^{59}$. The electron and hole effective masses were computed using parabolic line fitting at the direct band edges. We used the SUMO code ${ }^{79}$ to perform the effective mass calculations. In case there are degenerate bands at the conduction or the valence band edge, the SUMO code computes effective masses by performing parabolic fitting on all these bands. We use the lightest effective masses to estimate the minimum EBE.

\section{Excited state calculations using GW-BSE}

The quasiparticle energies were obtained by using many-body perturbations theory within both $\mathrm{G}_{0} \mathrm{~W}_{0}$ and $\mathrm{GW}_{0}$ approximation for the self-energy operator $29,31,38$. The basis set size for the response functions and $W$ was chosen to include all plane waves up to an energy cutoff of $340 \mathrm{eV}$. The number of unoccupied bands included in the GW calculation was selected such that the QP gap is converged to within $0.1 \mathrm{eV}$. The excitonic effects were studied by solving Bethe-Salpeter equation within Tamm-Dancoff approximation ${ }^{33,80}$. We included all the occupied/unoccupied bands which were within $1.5 \mathrm{eV}$ from band edges in the BSE calculation to ensure convergence of the absorption spectra. However, the convergence of the BSE absorption spectra with the number of $k$-points $\left(N_{k}^{\mathrm{BSE}}\right)$ is often quite challenging and computationally expensive ${ }^{33}$. Nonetheless, in this study we selected the $N_{k}^{\mathrm{BSE}}$ such that the EBE converged to within $0.1 \mathrm{eV}$. See Supplementary Table 6 for a list of all the converged parameters for each of the 48 materials.

\section{High-throughput workflow}

We have developed a Python code for performing automated firstprinciples calculations within GW-BSE framework and used it carry out all the calculations reported in this article. Our code is built upon open-source Python packages developed by the Materials Project, such as pymatgen ${ }^{81}$, fireWorks ${ }^{82}$, and atomate ${ }^{83}$ to achieve complete automation of the entire multi-step GW-BSE computational framework that requires several convergence parameters. GW-BSE calculation is extremly sensitive to multiple interdependent convergence parameters such as number of bands $\left(N_{\text {bands }}\right)$ included in the GW self-energy calculation, number of k-points $\left(\mathrm{N}_{k}^{B S}\right)$ used to sample the Brillouin zone in the BSE calculation etc. Our workflow is capable of performing automated convergence test of all these parameters and using those values to carry out fully converged GWBSE calculations. However, due to limitation of the computational resources available, we have restricted ourselves with converging $N_{\text {bands }}$ and $N_{k}^{B S E}$, two most sensitive parameters in the GW-BSE calculation. For several other convergence parameters such as, number of (imaginary) frequency grid points in GW calculation, energy cutoff for response function we have chosen a value large enough to produce quasiparticle energies within $0.1 \mathrm{eV}$. To reduce the computational cost associated with performing convergence tests we have judiciously used COHSEX and DFT level calculations which are significantly cheaper than full GW-BSE calculation. Details about these choices to perform efficient convergence calculations for GW-BSE calculations will be included in a forthcoming manuscript. In this workflow, we have also employed Wannier $90^{84}$, a program for calculating maximally-localized Wannier functions to perform the interpolation required to obtain quasiparticle bandstructure.

\section{DATA AVAILABILITY}

All data supporting the findings of this work are available in the article and its Supplementary Information. Extra data and machine readable data are available upon reasonable request to the authors.

\section{CODE AVAILABILITY}

The high-throughput GW-BSE workflow code used in this study are available from the authors upon reasonable request.

Received: 20 March 2021; Accepted: 24 September 2021; Published online: 19 November 2021

\section{REFERENCES}

1. Giebink, N. C., Wiederrecht, G. P., Wasielewski, M. R. \& Forrest, S. R. Thermodynamic efficiency limit of excitonic solar cells. Phys. Rev. B 83, 195326 (2011).

2. Wolfe, J. P. Thermodynamics of excitons in semiconductors. Phys. Today 35 , 46-54 (1982).

3. Meng, A., Zhang, L., Cheng, B. \& $\mathrm{Yu}, \mathrm{J}^{\mathrm{TiO}} \mathrm{T}_{2}-\mathrm{MnO}_{\mathrm{x}}-\mathrm{Pt}$ hybrid multiheterojunction film photocatalyst with enhanced photocatalytic $\mathrm{CO}_{2}$-reduction activity. ACS Appl. Mater. Interfaces 11, 5581-5589 (2018).

4. Dimroth, F. \& Kurtz, S. High-efficiency multijunction solar cells. MRS Bull. 32, 230-235 (2007).

5. Shockley, W. \& Queisser, H. J. Detailed balance limit of efficiency of p-n junction solar cells. J. Appl. Phys. 32, 510-519 (1961). 
6. Hashemi, S. M. H., Choi, J.-W. \& Psaltis, D. Solar thermal harvesting for enhanced photocatalytic reactions. Phys. Chem. Chem. Phys. 16, 5137-5141 (2014)

7. Shockley, W. \& Read Jr, W. Statistics of the recombinations of holes and electrons. Phys. Rev. 87, 835 (1952).

8. Richter, A., Hermle, M. \& Glunz, S. W. Reassessment of the limiting efficiency for crystalline silicon solar cells. IEEE J. Photovolt. 3, 1184-1191 (2013).

9. Auger, P. Sur les rayons $\beta$ secondaires produits dans un gaz par des rayons $\mathrm{x} . C R$ Acad. Sci. 177, 169 (1923).

10. Beattie, A. \& Landsberg, P. Auger effect in semiconductors. Proc. R. Soc. Lond. Ser. A. Math. Phys. Sci. 249, 16-29 (1959).

11. Vossier, A., Hirsch, B. \& Gordon, J. M. Is auger recombination the ultimate performance limiter in concentrator solar cells? Appl. Phys. Lett. 97, 193509 (2010).

12. Yang, $\mathrm{Y}$. et al. Comparison of recombination dynamics in $\mathrm{CH}_{3} \mathrm{NH}_{3} \mathrm{PbBr}_{3}$ and $\mathrm{CH}_{3} \mathrm{NH}_{3} \mathrm{Pbl}_{3}$ perovskite films: influence of exciton binding energy. J. Phys. Chem. Lett. 6, 4688-4692 (2015).

13. Wang, F., Wu, Y., Hybertsen, M. S. \& Heinz, T. F. Auger recombination of excitons in one-dimensional systems. Phys. Rev. B 73, 245424 (2006).

14. Wang, $H$. et al. Insights into the excitonic processes in polymeric photocatalysts. Chem. Sci. 8, 4087-4092 (2017).

15. Guo, Z., Zhou, J., Zhu, L. \& Sun, Z. Mxene: a promising photocatalyst for water splitting. J. Mater. Chem. A 4, 11446-11452 (2016).

16. Shinde, A. et al. Discovery of manganese-based solar fuel photoanodes via integration of electronic structure calculations, pourbaix stability modeling, and high-throughput experiments. ACS Energy Lett. 2, 2307-2312 (2017).

17. Yan, Q. et al. Solar fuels photoanode materials discovery by integrating highthroughput theory and experiment. Proc. Natl Acad. Sci. 114, 3040-3043 (2017).

18. Singh, A. K., Montoya, J. H., Gregoire, J. M. \& Persson, K. A. Robust and synthesizable photocatalysts for $\mathrm{CO}_{2}$ reduction: a data-driven materials discovery. Nat. Commun. 10, 1-9 (2019).

19. Singh, A. K. et al. Electrochemical stability of metastable materials. Chem. Mater. 29, 10159-10167 (2017).

20. Umari, P., Mosconi, E. \& De Angelis, F. Relativistic GW calculations on $\mathrm{CH}_{3} \mathrm{NH}_{3} \mathrm{Pbl}_{3}$ and $\mathrm{CH}_{3} \mathrm{NH}_{3} \mathrm{Snl}_{3}$ perovskites for solar cell applications. Sci. Rep. 4, 1-7 (2014).

21. Landmann, M., Rauls, E. \& Schmidt, W. The electronic structure and optical response of rutile, anatase and brookite $\mathrm{TiO}_{2}$. J. Phys. Condens. Matter 24, 195503 (2012).

22. Schmidt, F. et al. Quasiparticle and excitonic effects in the optical response of $\mathrm{KNbO}_{3}$. Phys. Rev. Mater. 3, 054401 (2019).

23. Tran, V., Soklaski, R., Liang, Y. \& Yang, L. Layer-controlled band gap and anisotropic excitons in few-layer black phosphorus. Phys. Rev. B 89, 235319 (2014).

24. Ugeda, M. M. et al. Giant bandgap renormalization and excitonic effects in a monolayer transition metal dichalcogenide semiconductor. Nat. Mater. 13, 1091-1095 (2014).

25. Qiu, D. Y., Felipe, H. \& Louie, S. G. Optical spectrum of $\mathrm{MoS}_{2}$ : many-body effects and diversity of exciton states. Phys. Rev. Lett. 111, 216805 (2013).

26. Shehzad, N., Tahir, M., Johari, K., Murugesan, T. \& Hussain, M. A critical review on $\mathrm{TiO}_{2}$ based photocatalytic $\mathrm{CO}_{2}$ reduction system: Strategies to improve efficiency. J. CO2 Utilization 26, 98-122 (2018).

27. Wu, Y., Lazic, P., Hautier, G., Persson, K. \& Ceder, G. First principles high throughput screening of oxynitrides for water-splitting photocatalysts. Energy Environ. Sci. 6, 157-168 (2013).

28. Yu, L. \& Zunger, A. Identification of potential photovoltaic absorbers based on first-principles spectroscopic screening of materials. Phys. Rev. Lett. 108, 068701 (2012).

29. Hedin, L. New method for calculating the one-particle green's function with application to the electron-gas problem. Phys. Rev. 139, A796 (1965).

30. Hybertsen, M. S. \& Louie, S. G. Electron correlation in semiconductors and insulators: band gaps and quasiparticle energies. Phys. Rev. B 34, 5390 (1986).

31. Shishkin, M. \& Kresse, G. Self-consistent GW calculations for semiconductors and insulators. Phys. Rev. B 75, 235102 (2007).

32. Viñes, F., Lamiel-García, O., Chul Ko, K., Yong Lee, J. \& Illas, F. Systematic study of the effect of HSE functional internal parameters on the electronic structure and band gap of a representative set of metal oxides. J. Comput. Chem. 38, 781-789 (2017).

33. Sander, T., Maggio, E. \& Kresse, G. Beyond the tamm-dancoff approximation for extended systems using exact diagonalization. Phys. Rev. B 92, 045209 (2015).

34. Lewis, D. K., Ramasubramaniam, A. \& Sharifzadeh, S. Tuned and screened rangeseparated hybrid density functional theory for describing electronic and optical properties of defective gallium nitride. Phys. Rev. Mater. 4, 063803 (2020).

35. Onida, G., Reining, L. \& Rubio, A. Electronic excitations: density-functional versus many-body green's-function approaches. Rev. Mod. Phys. 74, 601 (2002).

36. Malone, B. D. \& Cohen, M. L. Quasiparticle semiconductor band structures including spin-orbit interactions. J. Phys. Condens. Matter 25, 105503 (2013).

37. Malashevich, A., Jain, M. \& Louie, S. G. First-principles DFT + GW study of oxygen vacancies in rutile $\mathrm{TiO}_{2}$. Phys. Rev. B 89, 075205 (2014).
38. Shishkin, M. \& Kresse, G. Implementation and performance of the frequencydependent GW method within the PAW framework. Phys. Rev. B 74, 035101 (2006).

39. Dion, M., Rydberg, H., Schröder, E., Langreth, D. C. \& Lundqvist, B. I. Van der waals density functional for general geometries. Phys. Rev. Lett. 92, 246401 (2004).

40. Klimeš, J., Bowler, D. R. \& Michaelides, A. Chemical accuracy for the van der waals density functional. J. Phys. Condens. Matter 22, 022201 (2009).

41. Belsky, A., Hellenbrandt, M., Karen, V. L. \& Luksch, P. New developments in the inorganic crystal structure database (ICSD): accessibility in support of materials research and design. Acta Crystallogr. Sect. B Struct. Sci. 58, 364-369 (2002).

42. van Schilfgaarde, M., Kotani, T. \& Faleev, S. Quasiparticle self-consistent GW theory. Phys. Rev. Lett. 96, 226402 (2006).

43. Baldini, E. et al. Strongly bound excitons in anatase $\mathrm{TiO}_{2}$ single crystals and nanoparticles. Nat. Commun. 8, 1-11 (2017).

44. Park, S. et al. Direct determination of monolayer $\mathrm{MoS}_{2}$ and $\mathrm{WSe}_{2}$ exciton binding energies on insulating and metallic substrates. 2D Mater. 5, 025003 (2018).

45. Zhang, X.-X., You, Y., Zhao, S. Y. F. \& Heinz, T. F. Experimental evidence for dark excitons in monolayer WSe 2 . Phys. Rev. Lett. 115, 257403 (2015).

46. Malic, E. et al. Dark excitons in transition metal dichalcogenides. Phys. Rev. Mater. 2, 014002 (2018)

47. Green, M. A. \& Keevers, M. J. Optical properties of intrinsic silicon at 300 K. Prog. Photovoltaics Res. Appl 3, 189-192 (1995).

48. Chiker, F., Kebbab, Z., Miloua, R. \& Benramdane, N. Birefringence of optically uniaxial ternary semiconductors. Solid State Commun. 151, 1568-1573 (2011).

49. Cheddadi, S. et al. First-principle calculations of structural, electronic, optical, elastic and thermal properties of $\mathrm{MgXAs}_{2(\mathrm{X}=\mathrm{Si}, \mathrm{Ge})}$ compounds. Pramana 89, 89 (2017).

50. Woolley, J. \& Ray, B. Effects of solid solution of $\mathrm{Ga}_{2} \mathrm{Te}_{3}$ with $\mathrm{A}_{\| \mid} \mathrm{B}_{\mathrm{V} \mid}$ tellurides. J. Phys. Chem. Solids 16, 102-106 (1960).

51. Wirtz, L., Marini, A. \& Rubio, A. Excitons in boron nitride nanotubes: dimensionality effects. Phys. Rev. Lett. 96, 126104 (2006).

52. Hsueh, H., Guo, G. \& Louie, S. G. Excitonic effects in the optical properties of a SiC sheet and nanotubes. Phys. Rev. B 84, 085404 (2011).

53. Cudazzo, P., Attaccalite, C., Tokatly, I. V. \& Rubio, A. Strong charge-transfer excitonic effects and the bose-einstein exciton condensate in graphane. Phys. Rev. Lett. 104, 226804 (2010).

54. Yang, L. Excitons in intrinsic and bilayer graphene. Phys. Rev. B 83, 085405 (2011).

55. Torrisi, S. B., Singh, A. K., Montoya, J. H., Biswas, T. \& Persson, K. A. Twodimensional forms of robust $\mathrm{CO}_{2}$ reduction photocatalysts. npj 2D Mater. Appl. 4, 1-10 (2020).

56. Singh, A. K. \& Hennig, R. G. Computational prediction of two-dimensional groupIV mono-chalcogenides. Appl. Phys. Lett. 105, 042103 (2014).

57. Paul, J. et al. Computational methods for 2D materials: discovery, property characterization, and application design. J. Phys.: Condens. Matter 29, 473001 (2017).

58. Wannier, G. H. The structure of electronic excitation levels in insulating crystals. Phys. Rev. 52, 191 (1937).

59. Gajdoš, M., Hummer, K., Kresse, G., Furthmüller, J. \& Bechstedt, F. Linear optical properties in the projector-augmented wave methodology. Phys. Rev. B 73 045112 (2006)

60. Weaver, K. F., Morales, V. C., Dunn, S. L., Godde, K. \& Weaver, P. F. An introduction to statistical analysis in research: with applications in the biological and life sciences (John Wiley \& Sons, 2017).

61. Wang, X. et al. A class of two-dimensional SiAs monolayers with novel electronic and optical properties from ab initio investigations. Eur. Phys. J. 134, 287 (2019).

62. Kunioka, A., Ho, K. \& Sakai, Y. Optical properties of SiAs single crystals. J. Appl. Phys. 44, 1895-1896 (1973).

63. Wing, D. et al. Comparing time-dependent density functional theory with manybody perturbation theory for semiconductors: Screened range-separated hybrids and the GW plus Bethe-Salpeter approach. Phys. Rev. Mater. 3, 064603 (2019).

64. Blunt, R., Frederikse, H., Becker, J. \& Hosler, W. Electrical and optical properties of intermetallic compounds. III. aluminum antimonide. Phys. Rev. 96, 578-580 (1954).

65. Antonius, G., Qiu, D. Y. \& Louie, S. G. Orbital symmetry and the optical response of single-layer MX monochalcogenides. Nano Lett. 18, 1925-1929 (2018).

66. Wadsten, T. The crystal structure of SiAs. Acta Chem. Scandinavica 19, 1232-1238 (1965).

67. Julien Pouzol, M., Jaulmes, S., Guittard, M. \& Alapini, F. Monotellurure de gallium, GaTe. Acta Crystallogr. B35, 2848-2851 (1979).

68. Kuehn, G., Schumann, B., Oppermann, D., Neumann, H. \& Sobotta, H. Preparation, structure, and infrared lattice vibrations of $\mathrm{LilnTe}_{2}$. Z. fuer Anorganische und Allg. Chem. (DE) 531, 61-66 (1985)

69. Errandonea, D. et al. X-ray diffraction study on pressure-induced phase transformations and the equation of state of $\mathrm{ZnGa}_{2} \mathrm{Te}_{4}$. J. Appl. Phys. 114 233507-1-233507-7 (2013). 
70. Neumann, H., Moise, E., Schwer, H. \& Kramer, V. Infrared lattice vibrations of $\mathrm{CdGa}_{2} \mathrm{Te}_{4}$. Cryst. Res. Technol. 28, 635-639 (1993).

71. Bendavid, L. I. \& Carter, E. A. Status in Calculating Electronic Excited States in Transition Metal Oxides from First Principles, in (eds C. Di Valentin, S. Botti \& M. Cococcioni) First Principles Approaches to Spectroscopic Properties of Complex Materials. Topics in Current Chemistry. https://doi.org/10.1007/128_2013_503 (Springer: Berlin, Heidelberg, 2014).

72. Bruneval, F. et al. Exchange and correlation effects in electronic excitations of $\mathrm{Cu}_{2}$ O. Phys. Rev. Lett. 97, 267601 (2006).

73. Biswas, T. \& Jain, M. Quasiparticle band structure and optical properties of hexagonal-YMnO . J. Appl. Phys. 120, 155102 (2016).

74. Kang, W. \& Hybertsen, M. S. Quasiparticle and optical properties of rutile and anatase $\mathrm{TiO}_{2}$. Phys. Rev. B 82, 085203 (2010).

75. Ganduglia-Pirovano, M. V., Hofmann, A. \& Sauer, J. Oxygen vacancies in transition metal and rare earth oxides: Current state of understanding and remaining challenges. Surf. Sci. Rep. 62, 219-270 (2007).

76. Kresse, G. \& Joubert, D. From ultrasoft pseudopotentials to the projector augmented-wave method. Phys. Rev. B 59, 1758 (1999).

77. Jain, A. et al. A high-throughput infrastructure for density functional theory calculations. Comput. Mater. Sci. 50, 2295-2310 (2011).

78. Baroni, S. \& Resta, R. Ab initio calculation of the macroscopic dielectric constant in silicon. Phys. Rev. B 33, 7017 (1986).

79. Ganose, A., Jackson, A. \& Scanlon, D. sumo: Command-line tools for plotting and analysis of periodic* ab initio* calculations. J. Open Source Softw. 3, 717 (2018).

80. Rohlfing, M. \& Louie, S. G. Electron-hole excitations and optical spectra from first principles. Phys. Rev. B 62, 4927 (2000).

81. Ong, S. P. et al. Python materials genomics (pymatgen): A robust, open-source python library for materials analysis. Comput. Mater. Sci. 68, 314-319 (2013).

82. Jain, A. et al. Fireworks: A dynamic workflow system designed for high-throughput applications. Concurrency Comput. Pract. Experience 27, 5037-5059 (2015).

83. Mathew, K. et al. Atomate: A high-level interface to generate, execute, and analyze computational materials science workflows. Comput. Mater. Sci. 139, 140-152 (2017).

84. Mostofi, A. A. et al. wannier90: A tool for obtaining maximally-localised wannier functions. Computer Phys. Commun. 178, 685-699 (2008).

85. Amtout, A. \& Leonelli, R. Optical properties of rutile near its fundamental band gap. Phys. Rev. B 51, 6842 (1995).

86. Tang, H., Levy, F., Berger, H. \& Schmid, P. Urbach tail of anatase $\mathrm{TiO}_{2}$. Phys. Rev. $B$ 52, 7771 (1995).

87. Tezuka, Y. et al. Photoemission and bremsstrahlung isochromat spectroscopy studies of $\mathrm{TiO}_{2}$ (rutile) and $\mathrm{SrTiO}_{3}$. J. Phys. Soc. Jpn. 63, 347-357 (1994).

88. Griffiths, T. R., Davies, M. J. \& Hubbard, H. V. S. A. Spectroscopic studies on single crystals having the fluorite lattice. part 1.-the fundamental absorption edge; urbach's rule and the debye temperature in $\mathrm{CeO}_{2}$. J. Chem. Soc., Faraday Trans. 2 Mol. Chem. Phys. 72, 765-772 (1976).

89. Kam, K. \& Parkinson, B. Detailed photocurrent spectroscopy of the semiconducting group vib transition metal dichalcogenides. J. Phys. Chem. 86, 463-467 (1982).

90. Pässler, R. et al. Temperature dependence of exciton peak energies in $\mathrm{ZnS}, \mathrm{ZnSe}$, and ZnTe epitaxial films. J. Appl. Phys. 86, 4403-4411 (1999).

91. Madelung, O. Semiconductors: group IV elements and III-V compounds (Springer Science \& Business Media, 2012).

\section{ACKNOWLEDGEMENTS}

This work was supported by the Arizona State University start-up funds and in part as part of ULTRA, an Energy Frontier Research Center funded by the U.S. Department of Energy (DOE), Office of Science, Basic Energy Sciences (BES), under Award \# DESC0021230 (GW-BSE high-throughput simulations). In addition, Singh acknowledges support by the NSF DMR-grant NSF-DMR \#1906030. The authors acknowledge the San Diego Supercomputer Center under the NSF-XSEDE Award No. DMR150006 and the Research Computing at Arizona State University for providing HPC resources. This research used resources of the National Energy Research Scientific Computing Center, a DOE Office of Science User Facility supported by the Office of Science of the U.S. Department of Energy under Contract No. DE-AC02-05CH11231.

\section{AUTHOR CONTRIBUTIONS}

T.B. performed the simulations and calculations. Both the authors contributed to designing the research methods, interpreting the data, and writing the manuscript.

\section{COMPETING INTERESTS}

The authors declare no competing interests.

\section{ADDITIONAL INFORMATION}

Supplementary information The online version contains supplementary material available at https://doi.org/10.1038/s41524-021-00640-3.

Correspondence and requests for materials should be addressed to Arunima K. Singh.

Reprints and permission information is available at http://www.nature.com/ reprints

Publisher's note Springer Nature remains neutral with regard to jurisdictional claims in published maps and institutional affiliations.

(c) Open Access This article is licensed under a Creative Commons Attribution 4.0 International License, which permits use, sharing, adaptation, distribution and reproduction in any medium or format, as long as you give appropriate credit to the original author(s) and the source, provide a link to the Creative Commons license, and indicate if changes were made. The images or other third party material in this article are included in the article's Creative Commons license, unless indicated otherwise in a credit line to the material. If material is not included in the article's Creative Commons license and your intended use is not permitted by statutory regulation or exceeds the permitted use, you will need to obtain permission directly from the copyright holder. To view a copy of this license, visit http://creativecommons. org/licenses/by/4.0/.

(c) The Author(s) 2021 